\title{
Structure of nucleotide-binding domain 1 of the cystic fibrosis transmembrane conductance regulator
}

\author{
Hal A Lewis ${ }^{1, *}$, Sean G Buchanan ${ }^{1}$, \\ Stephen K Burley ${ }^{1}$, Kris Conners ${ }^{1}$, Mark \\ Dickey ${ }^{1}$, Michael Dorwart ${ }^{2}$, Richard \\ Fowler $^{1}$, Xia Gao ${ }^{1}$, William B Guggino ${ }^{3}$, \\ Wayne A Hendrickson ${ }^{4}$, John F Hunt ${ }^{5}$, \\ Margaret C Kearins ${ }^{1}$, Don Lorimer ${ }^{1}$, Peter C \\ Maloney $^{3}$, Kai W Post ${ }^{1}$, Kanagalaghatta R \\ Rajashankar', Marc E Rutter ${ }^{1}$, J Michael \\ Sauder ${ }^{1}$, Stephanie Shriver ${ }^{1}$, Patrick H \\ Thibodeau ${ }^{2}$, Philip J Thomas ${ }^{2}$, Marie \\ Zhang ${ }^{1}$, Xun Zhao' and Spencer Emtage ${ }^{1}$
}

${ }^{1}$ Structural GenomiX Inc., San Diego, CA, USA, ${ }^{2}$ Department of Physiology, University of Texas Southwestern Medical Center, Dallas, TX, USA, ${ }^{3}$ Department of Physiology, School of Medicine, The Johns Hopkins University, Baltimore, MD, USA, ${ }^{4}$ Department of Biochemistry and Molecular Biophysics, Howard Hughes Medical Institute, Columbia University, New York, NY, USA and ${ }^{5}$ Department of Biological Sciences, Columbia University, New York, NY, USA

Cystic fibrosis transmembrane conductance regulator (CFTR) is an ATP-binding cassette (ABC) transporter that functions as a chloride channel. Nucleotide-binding domain 1 (NBD1), one of two ABC domains in CFTR, also contains sites for the predominant CF-causing mutation and, potentially, for regulatory phosphorylation. We have determined crystal structures for mouse NBD1 in unliganded, ADP- and ATP-bound states, with and without phosphorylation. This NBD1 differs from typical ABC domains in having added regulatory segments, a foreshortened subdomain interconnection, and an unusual nucleotide conformation. Moreover, isolated NBD1 has undetectable ATPase activity and its structure is essentially the same independent of ligand state. Phe508, which is commonly deleted in $\mathrm{CF}$, is exposed at a putative NBD1transmembrane interface. Our results are consistent with a CFTR mechanism, whereby channel gating occurs through ATP binding in an NBD1-NBD2 nucleotide sandwich that forms upon displacement of NBD1 regulatory segments.

The EMBO Journal (2004) 23, 282-293. doi:10.1038/

sj.emboj.7600040; Published online 18 December 2003

Subject Categories: structural biology; molecular biology of disease

Keywords: ABC transporter; CFTR; crystal structure; NBD1

\footnotetext{
*Corresponding author. Structural GenomiX Inc., 10505 Roselle St., San Diego, CA 92121, USA. Tel.: + 1858228 1555; Fax: + 1858457 4533; E-mail: hal_lewis@stromix.com
}

Received: 23 September 2003; accepted: 25 November 2003; Published online: 18 December 2003

\section{Introduction}

Cystic fibrosis (CF) is the most prevalent lethal, autosomalrecessive genetic disease among Caucasians. CF patients have severely reduced life expectancies, largely because of chronic pulmonary damage. The root cause of CF is in defective cystic fibrosis transmembrane conductance regulator (CFTR) (Riordan et al, 1989). Human CFTR is a 1480 residue, multidomain, integral membrane protein that regulates chloride ion flow across the cell membrane. It is a member of the ATPbinding cassette $(\mathrm{ABC})$ transporter superfamily of proteins and consists of two membrane-spanning domains (MSDs), two nucleotide-binding domains (NBDs), and a regulatory region (R) arranged in the order MSD1-NBD1-R-MSD2NBD2 (Figure 1). The NBDs of ABC transporters are typified by a consensus ATP-binding region, which encompasses two Walker motifs (A and B regions), a highly conserved region called the signature sequence (LSGGQ), plus other conserved functional features identified as the Q- and H-loops, named respectively for glutamine and histidine residues involved in ATP recognition and hydrolysis. The most common CF mutation is the deletion of CFTR phenylalanine 508 ( $\Delta \mathrm{F} 508)$, which is located in NBD1. In total, $70 \%$ of CF alleles have $\Delta \mathrm{F} 508$ and $90 \%$ of CF patients have at least one copy of this deletion. A better understanding of the structure and function of NBD1 and the role of Phe508 may accelerate the development of new approaches to the treatment of CF.

Atomic-level structural information has been obtained for components of several $\mathrm{ABC}$ transporter systems. Complete bacterial transmembrane transporter proteins MsbA (Chang and Roth, 2001; Chang, 2003) and BtuCD (Locher et al, 2002) have been analyzed at modest resolution, showing similar associations between NBD and MSD domains but markedly different overall architectures. High-resolution X-ray structures have also been determined for several prokaryotic NBDs (Schmitt and Tampe, 2002), most recently HlyB (Schmitt et al, 2003) and GlcV (Verdon et al, 2003), and for one eukaryotic NBD, TAP1 (Gaudet and Wiley, 2001). All such NBDs have a common fold characterized by two subdomains: one contains an F1-like ATP-binding core plus an ABC-specific antiparallel $\beta$ region and the other an ABC-specific $\alpha$-helical domain (Karpowich et al, 2001). The F1-like portion contains the primary determinants of nucleotide binding; the antiparallel $\beta$ portion adds interactions to the base and ribose groups; and the ABC signature sequence of the $\alpha$-helical domain from a dimer mate completes productive coordination of the ATP $\beta$ - and $\gamma$-phosphate groups of the nucleotide (Figure 1 ).

Typical ABC transporters are thought to function as labile dimers in which coupling of ATP hydrolysis to movements in transmembrane segments drives the translocation of relevant entities across the membrane. The Rad50 DNA repair enzyme, a remote homolog of $\mathrm{ABC}$ proteins, provided the first structural model for the dimer state (Hopfner et al, 2000), and similar dimers form in BtuCD (Locher et al, 2002) and in a catalytically impaired E171Q variant of 


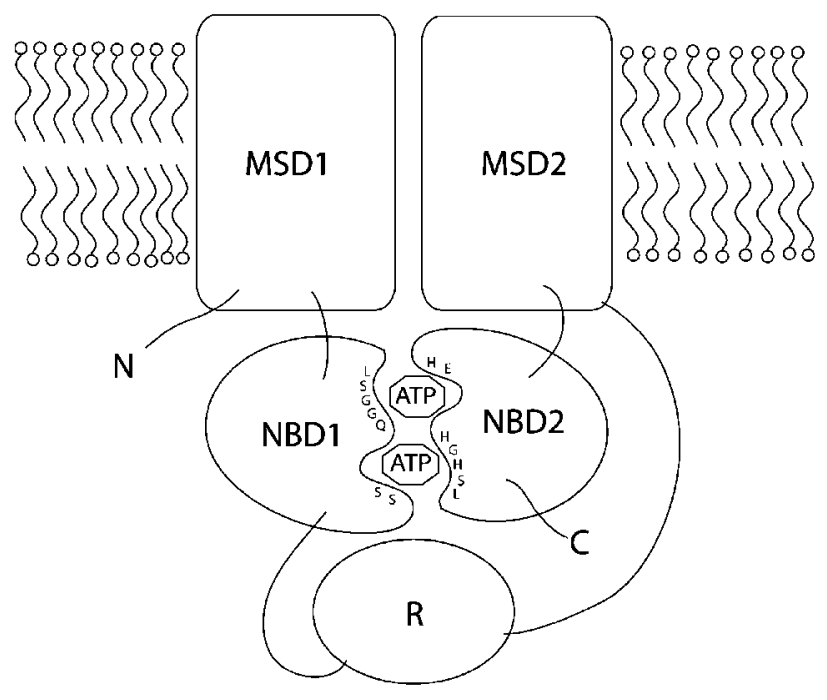

Figure 1 Domain organization of CFTR. The five domains of CFTR are shown. Also indicated is a putative nucleotide-binding domain association in which the ATP-binding site of one NBD is opposed by the signature sequence of the other NBD. Inactivity at the NBD1 ATP-binding site is indicated by Ser residues in place of the catalytic Glu and His in addition to His residues substituted for the Gln and central Gly in the NBD2 signature sequence.

MJ0796 when complexed with ATP (Smith et al, 2002). The nucleotides in this symmetric dimer are sandwiched at the interface between protomers such that the LSGGQ residues from one complete the binding interactions of nucleotide in the apposing protomer. The putative functional NBD 'dimer' in CFTR is believed to be intramolecular and necessarily asymmetric since 'hemichannel' constructs produced wildtype activity when expressed together but not separately (Ostedgaard et al, 1997; Chan et al, 2000).

The boundaries of CFTR NBD1 have been a matter of some controversy. It has been suggested to begin in the span from residue 373 (Wang et al, 2002) to residue 441 (Bianchet et al, 1997) at the N-terminus and to end in the span from residue 586 (Riordan et al, 1989) to residue 684 (Bianchet et al, 1997) at the C-terminus (human CFTR numbering, used throughout this paper). A compelling functional definition based on the coexpression of severed and deleted CFTR constructs gave boundaries within 433-633 for NBD1 (Chan et al, 2000). Many different expression constructs have been used in studies of NBD1 function, sometimes yielding conflicting results. Indeed, several aspects of CFTR NBD1 function remain poorly characterized. The most important outstanding issues pertain to ATP binding and hydrolysis, conformational adaptability in the domain, the effects of NBD1 phosphorylation, and the structural consequences of disease-causing mutations, in particular $\Delta \mathrm{F} 508$. We have determined the crystal structure of murine NBD1 to shed light on these vital mechanistic issues.

\section{Results}

\section{mNBD1 expression and purification}

Given the uncertainty in the globular domain definition of CFTR NBD1, we chose to clone and express in Escherichia coli many constructs in parallel covering residues from 363 to 686. A pan-genomic approach was employed, testing the solubility of such NBD1 constructs from 10 organisms (human, mouse, baboon, macaque, sheep, rabbit, frog, salmon, killifish, and dogfish). The high-level production of soluble proteins $(>5 \mathrm{mg} / \mathrm{l})$ that are nonaggregated in solution was limited to a narrow globular domain definition (N-terminus: residues 385-391; C-terminus: residues 670680). Optimal recombinant protein was obtained from mouse CFTR with an expression construct spanning residues 389-673. Attempts with human NBD1 have not yet succeeded. We refer to the resulting mouse proteins as mNBD1 and mNBD1-P for the unphosphorylated and phosphorylated forms, respectively. Dynamic light scattering (DLS) and analytical gel filtration chromatography (GFC) measurements indicated that purified, recombinant mNBD1 was both monodisperse and monomeric. This protein was used for all of the structural studies reported here. A mutated version, K464A, which was expected to have reduced ATP binding, was also cloned and purified in the same manner and was used for ATP-binding measurements (see below).

\section{mNBD1 crystallization and structure determination}

Both native (S-Met) and selenomethionyl (Se-Met) mNBD1 crystallized in two morphologies under similar conditions: parallelepipeds in space group $\mathrm{P}_{42} 2$ and tetragonal bipyramids in space group $14_{1} 22$. The structure of mNBD1 bound to Mg-AMP.PNP was initially determined by multiple isomorphous replacement with anomalous scattering (MIRAS) phasing from $\mathrm{Ta}_{6} \mathrm{Br}_{12}$-soaked $\mathrm{I}_{1} 22$ crystals. This was subsequently improved using single-wavelength anomalous diffraction (SAD) data from Se-Met mNBD1 P42 2 crystals. Most of the protein sequence could be built into the SAD-generated experimental electron density map, the primary exception being residues 413-428, which were not well visualized (exact boundaries are given in Table I for each refined molecule). The initial structure of mNBD1 was subsequently used to solve the $14_{1} 22$ structure in the presence of Mg-ATP by molecular replacement and to determine isomorphous $\mathrm{P}_{2} 2_{1} 2$ structures in the presence of Mg-ATP, Mg-ADP, and in the absence of nucleotide. The conformation of mNBD1 is essentially the same in all crystal forms irrespective of the identity of the bound nucleotide and also in each of the four copies in the $P 422_{1} 2$ crystals and two copies in the I4 122 crystal. Each mNBD1 molecule is associated in a fourfold symmetric, head-to-tail ring structure that recurs three times in the $\mathrm{P} 42_{1} 2$ lattice and once in the $\mathrm{I}_{1} 22$ lattice.

\section{Overall structure of CFTR NBD1}

The mNBD1 domain has a core tertiary structure similar to NBDs from other ABC transporters, but this core is modified with major additions and deletions. Figure $2 \mathrm{~B}$ shows $\mathrm{a}$ topology diagram of mNBD1, indicating through color coding the subdomains and those regions of mNBD1 that show significant differences from other $A B C$ structures. Secondary structural elements in common with most known $\mathrm{ABC}$ structures are given conventional designations (S1, S2, S3, H1, etc.) and additional elements found in mNBD1 are denoted with lowercase letters (H1b, H1c, S6b, etc.). The three-dimensional course of the polypeptide chain is shown as a ribbon diagram in Figure $3 \mathrm{~A}$, where the structural elements are colored in the same code as in Figure $2 \mathrm{~B}$ and key elements in the binding of ATP are also identified. Figure $3 \mathrm{~B}$ shows a worm diagram of mNBD1 in 
Table I Data collection and refinement statistics

\begin{tabular}{|c|c|c|c|c|c|c|c|}
\hline & Space group & Resolution ( $(\AA)$ & Nucleotide & $\begin{array}{l}\text { Completeness (\%) } \\
\text { (overall/outer shell) }\end{array}$ & $\begin{array}{c}R_{\text {sym }}(\%) \\
\text { (overall/outer shell) }\end{array}$ & $\begin{array}{c}\text { Redundancy } \\
\text { (overall/outer shell) }\end{array}$ & $\begin{array}{c}I / \sigma(I) \\
\text { (overall/outer shell) }\end{array}$ \\
\hline $\begin{array}{l}\text { mNBD1 data collection } \\
\text { mNBD1 + AMP.PNP } \\
\text { mNBD1-P + ATP } \\
\text { mNBD1 apo } \\
\text { mNBD1 + ATP } \\
\text { mNBD1 + ADP } \\
\text { mNBD1 + ATP (2) }\end{array}$ & $\begin{array}{l}\text { P42 } 22 \\
\text { P42 } 22 \\
\text { P42 } 22 \\
\text { P42 } 22 \\
\text { P42 } 22 \\
\text { I4 } 222\end{array}$ & $\begin{array}{l}33.0-2.50 \\
24.0-2.35 \\
38.9-2.20 \\
39.2-2.20 \\
36.5-2.55 \\
49.7-3.00\end{array}$ & $\begin{array}{l}\text { AMP.PNP } \\
\text { ATP } \\
\text { None } \\
\text { ATP } \\
\text { ADP } \\
\text { ATP }\end{array}$ & $\begin{array}{c}99.9 / 99.6 \\
97.6 / 92.4 \\
93.2 / 95.1 \\
98.5 / 92.7 \\
100.0 / 99.4 \\
100.0 / 100.0\end{array}$ & $\begin{array}{r}12.2 / 35 \\
9.0 / 46 \\
7.6 / 44 \\
7.3 / 50 \\
7.7 / 35 \\
7.3 / 98\end{array}$ & $\begin{array}{c}14.1 / 13.6 \\
4.6 / 2.4 \\
5.1 / 4.1 \\
7.0 / 5.7 \\
9.8 / 9.9 \\
8.2 / 8.3\end{array}$ & $\begin{array}{r}5.4 / 1.8 \\
14.7 / 2.1 \\
19.8 / 3.1 \\
27.7 / 4.0 \\
8.0 / 1.9 \\
27.6 / 2.0\end{array}$ \\
\hline mNBD1 + ATP (2) & Resolution $(\AA)$ & $R$ & $R_{\text {free }}$ & Waters & $\begin{array}{l}\text { RMSD bond length } \\
(\AA)\end{array}$ & $\begin{array}{l}\text { RMSD bond angles } \\
{ }_{\left({ }^{\circ}\right)}\end{array}$ & $\underset{\left(\AA^{2}\right)}{\text { Average } B \text {-factors }}$ \\
\hline $\begin{array}{l}\text { Refinement statistics } \\
\text { mNBD1 + AMP.PNP }(1 \mathrm{Q} 3 \mathrm{H}) \\
\text { mNBD1-P + ATP (1ROZ) } \\
\text { mNBD1 apo (1ROW) } \\
\text { mNBD1 + ATP (1ROX) } \\
\text { mNBD + ADP (1ROY) } \\
\text { mNBD1 + ATP (2) (1R10) }\end{array}$ & $\begin{array}{l}33.0-2.50 \\
24.0-2.35 \\
38.9-2.20 \\
39.2-2.20 \\
36.0-2.55 \\
30.0-3.00\end{array}$ & $\begin{array}{l}0.215 \\
0.221 \\
0.231 \\
0.234 \\
0.207 \\
0.228\end{array}$ & $\begin{array}{l}0.266 \\
0.258 \\
0.262 \\
0.266 \\
0.257 \\
0.265\end{array}$ & $\begin{array}{r}658 \\
304 \\
423 \\
378 \\
195 \\
0\end{array}$ & $\begin{array}{l}0.008 \\
0.015 \\
0.021 \\
0.021 \\
0.015 \\
0.012\end{array}$ & $\begin{array}{l}1.9 \\
1.5 \\
2.0 \\
2.0 \\
1.7 \\
1.3\end{array}$ & $\begin{array}{l}36.4 \\
48.0 \\
30.8 \\
39.1 \\
45.6 \\
80.6\end{array}$ \\
\hline \multirow{2}{*}{ mNBD1 + ATP (2) (1R10) } & & & & & \multicolumn{3}{|c|}{ Ramachandran distribution } \\
\hline & Molecule A & Molecule B & Molecule C & Molecule D & Core & Allowed & Disallowed \\
\hline $\begin{array}{l}\text { Residues modeled } \\
\text { mNBD1 + AMP.PNP }\end{array}$ & $390-412,429-670$ & $389-412,429-670$ & $388-412,429-670$ & $\begin{array}{l}390-412 \\
429-670\end{array}$ & $92.0 \%$ & $7.9 \%$ & $0.0 \%$ \\
\hline mNBD1-P + ATP & $390-413,420-670$ & $389-412,420-670$ & $388-413,420-670$ & $390-407,430-670$ & $91.2 \%$ & $8.7 \%$ & $0.1 \%$ \\
\hline mNBD1 apo & $390-413,429-670$ & 389-413, 429-670 & $388-413,430-670$ & $390-412,430-670$ & $92.4 \%$ & $7.5 \%$ & $0.1 \%$ \\
\hline mNBD1 + ATP & $390-411,429-670$ & $389-411,429-670$ & $388-411,430-670$ & $390-411,430-670$ & $91.2 \%$ & $8.7 \%$ & $0.0 \%$ \\
\hline mNBD1 + ADP & $388-413,429-671$ & $388-411,428-670$ & $388-412,430-670$ & $390-412,430-671$ & $89.9 \%$ & $9.9 \%$ & $0.1 \%$ \\
\hline mNBD1 + ATP (2) & $391-412,429-670$ & $391-412,429-670$ & - & - & $85.5 \%$ & $14.5 \%$ & $0.0 \%$ \\
\hline
\end{tabular}

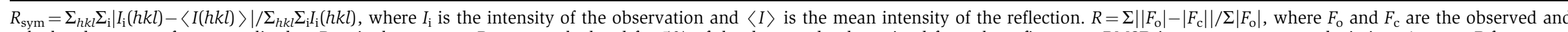

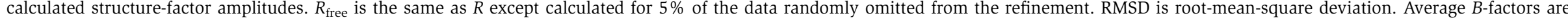
reported for all nonhydrogen atoms. The values in parenthesis following the dataset names are the Protein Data Bank identifiers for the respective structures. 
A

Numbering (H
mNBD1
hNBD1
mNBD2
hNBD2
pgp1-nt-Hs
pgp1-ct-Hs
mrp1-nt-Hs
MJ0796
HisP
TAP1

WalkerA

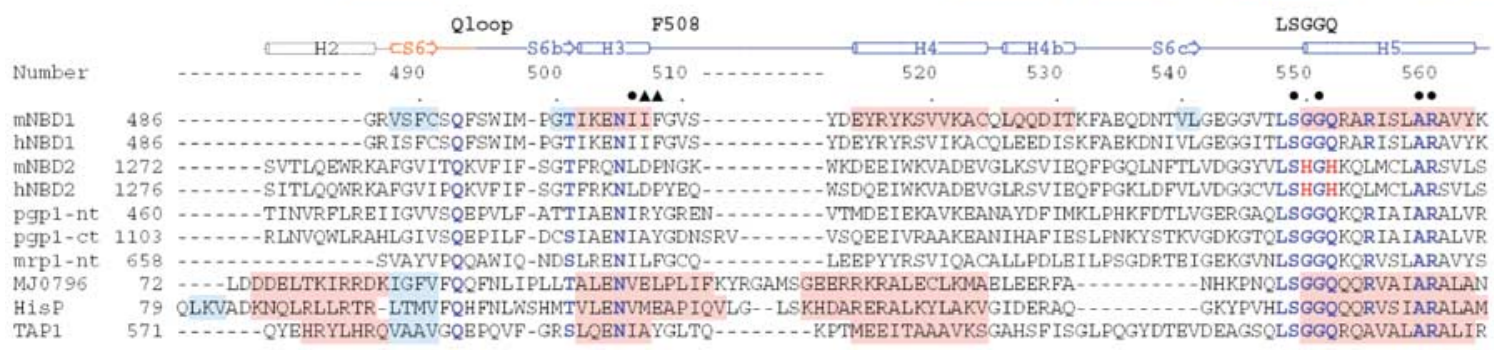

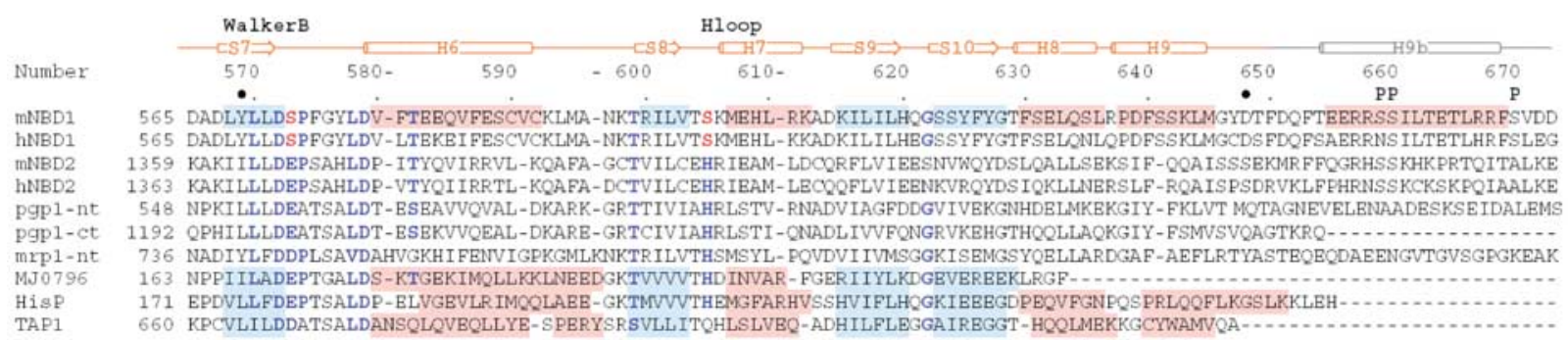

B

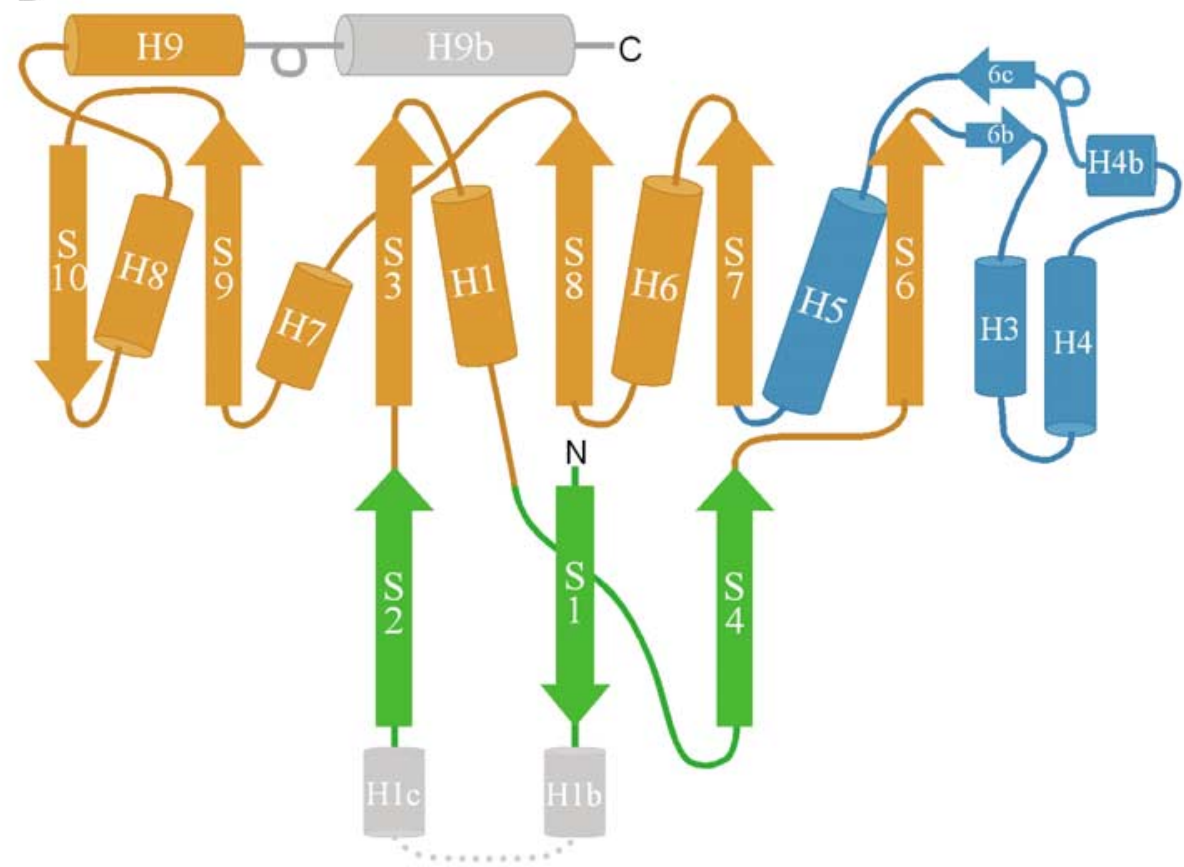

Figure 2 Sequence alignment and topology diagram of mNBD1. (A) Sequence alignment of human and mouse NBD1 and NBD2 with NBD domains from other ABC transporters. A blue background indicates $\beta$-strands while pink indicates $\alpha$-helices in known structures. Solid circles mark the locations of common CF-causative mutations, solid triangles the locations of deletion mutations, and $\mathrm{P}$ indicates where phosphorylation by PKA was observed in the mNBD1-P structure. Residues with high sequence conservation in ABC domains are highlighted in blue bold font. Red bold font indicates residues that significantly vary from this conservation. The secondary structure of mNBD1 is indicated graphically above the residue numbering row and is color coded by subdomain as in Figure 2B. (B) Topology diagram of mNBD1. The F1-type ATP-binding core subdomain is shown in gold, the ABC $\alpha$-subdomain in cyan, and the ABC $\beta$-subdomain in green. Regions of mNBD1 that are different from previous $\mathrm{ABC}$ structures are shown in gray. Circles indicate the positions of $3_{10}$ helices. 

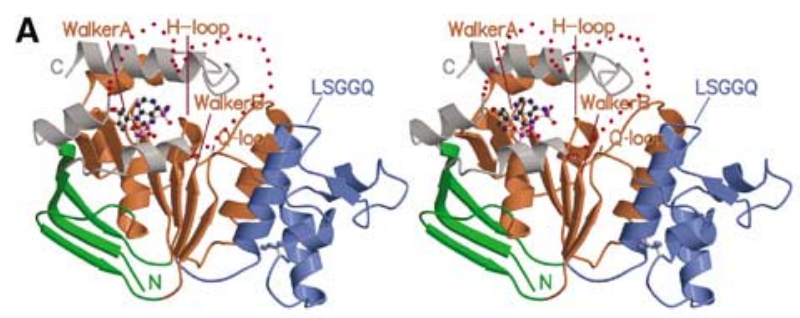

B

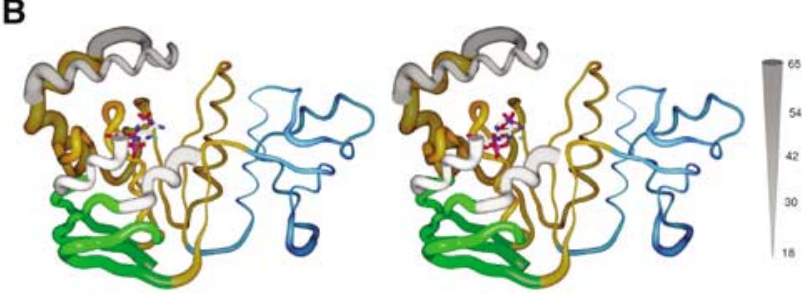

c
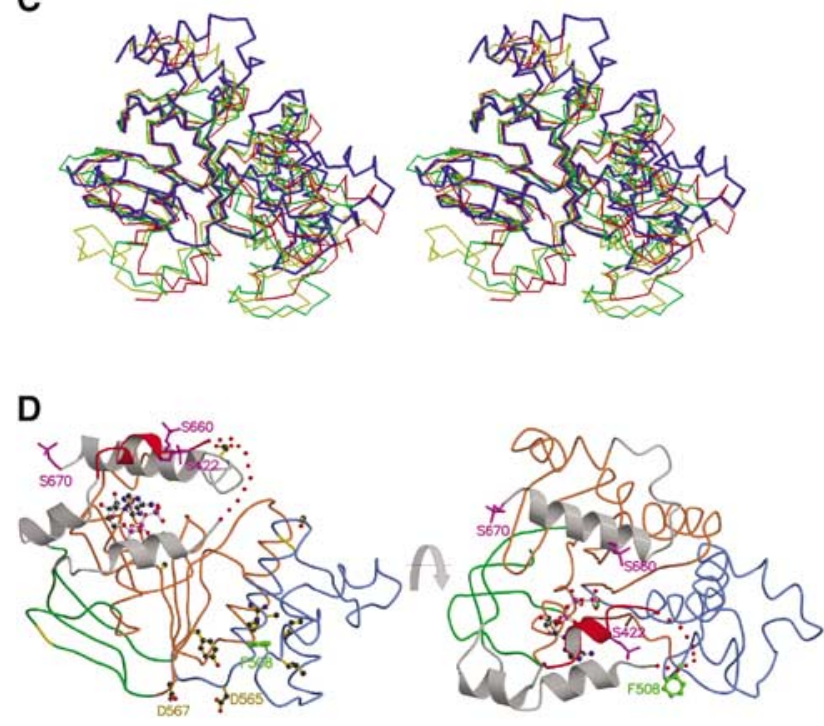

Figure 3 Structural fold of mNBD1. (A) Stereo ribbon diagram of mNBD1. ATP is shown in ball and stick representation. The subdomains are color coded as in Figure 2B. The dotted red line indicates residues missing from the mNBD1 model. (B) Stereo worm diagram of mNBD1. The worm thickness is indicative of the relative B-factor of the residues ranging from $18 \AA^{2}$ (thinnest) to $65 \AA^{2}$ (thickest). Color coding of subdomains is according to Figure $2 \mathrm{~B}$. (C) Stereo $\mathrm{C}_{\alpha}$ diagram of the superposition of representative ABC domain structures onto mNBD1. TAP1 (thin red), MJ0796 (thin green), and HisP (thin yellow) structures superimposed onto mNBD1 (thick blue). Superposition based on leastsquares alignments of the F1-type core and ABC-specific antiparallel $\beta$-subdomains. (D) Backbone structure of mNBD1 illustrating positions of phosphorylation and CF-causative mutations. Left: mNBD1 is seen in the same orientation and subdomain colorization as in Figure 3A. Right: the same structure rotated $80^{\circ}$ toward the viewer. Helices of regulatory segments are drawn as ribbons; the remaining polypeptide chain is a worm drawing. ATP is shown as ball and stick. Ser422, Ser659, Ser660, and Ser670 side chains are shown in purple. Residues 420-428 become ordered upon phosphorylation (solid red). The remaining residues of the structure that were not modeled (414-419) are indicated as red dots. Side chains are shown at sites of common CF-causative mutations (Ala455, Gly480, Ile506, Ile507, Ser549, Gly551, Ala559, Arg560, Tyr569, and Asp648 colored yellow; Phe508 in green). The di-acidic code residues (D565 and D567) are in gold. which the thickness of the trace is proportional to the Bfactors of the $\mathrm{C}_{\alpha}$ atoms, thereby reflecting potential mobility of the polypeptide backbone. Regions of highest mobility are near the $\mathrm{N}$ - and $\mathrm{C}$-termini and at the inserted and partially disordered segment between S1 and S2. B-factors are commonly elevated near termini and in some loops, but there may be special relevance here in relation to the putative NBD1/NBD2 interface (see below).

The core structure of mNBD1-ATP most closely resembles that of TAP1-ADP with a root-mean-square deviation (r.m.s.d.) of $1.9 \AA$ for $176 \mathrm{C}_{\alpha}$ atoms spanning a region with $26 \%$ sequence identity. MJ0796-ATP is next closest overall (2.4 $\AA$ r.m.s.d. over the same span; $30 \%$ sequence identity). This alignment omits the regions between $\mathrm{S} 1$ and $\mathrm{S} 2$, between S4 and S6, and beyond S10, which exhibit conformation differences between $\mathrm{ABC}$ structures. Figure $2 \mathrm{~A}$ presents a structure-based sequence alignment of $\mathrm{mNBD} 1$ with the $\mathrm{ABC}$ domains of some known structures and with selected sequences from human $\mathrm{ABC}$ transporters, including human CFTR. These comparisons should help to clarify the discussions of CFTR function.

Four major structural features distinguish mNBD1 from other ABC NBDs. This is evident in Figure 3C, where mNBD1 is superimposed onto three representative $A B C$ structures. First, mNBD1 contains an insertion of about 35 residues between $\beta$-strands S1 and S2. This insertion is composed of two short $\alpha$-helices (denoted H1b and H1c) separated by a flexible linker region that was not observed in the electron density map (residues 413-428, red dotted line in Figure 3A). The $\mathrm{N}$-terminal $\beta$-strand $\mathrm{S} 1$ is common to all $\mathrm{ABC}$ domain structures, and a conserved aromatic residue near its C-terminus (corresponding to Trp401 in CFTR) stacks against the adenine base in previously reported structures of $A B C$ domains complexed with nucleotides. The insertion leads to an altered binding geometry for the base and ribose in mNBD1 and includes a segment (415-432) that can be deleted while preserving function (Chan et al, 2000). Second, mNBD1 lacks a 14-27 residue region between $\beta$-strands S4 and S6 (residues 485-488) that typically contains an additional $\beta$-strand and an $\alpha$-helix (denoted S5 and $\mathrm{H} 2$ in Figure 2A; located lower left in Figure 3C). Third, relative to the prokaryotic $\mathrm{ABC}$ structures, mNBD1 is truncated between helices $\mathrm{H} 3$ and $\mathrm{H} 4$ (lower right of Figure 3C). Finally, the C-terminus of mNBD1 includes a long $\alpha$-helix (H9b) that is not present in other ABC domain structures. The hydrophobic face of this amphipathic $\alpha$-helix packs against a hydrophobic surface formed primarily by the preceding $\alpha$-helix H9. The H9b segment of the polypeptide chain has been considered to be part of the $\mathrm{R}$ domain as it contains two potentially regulatory phosphorylation sites, Ser660 and Ser670 (Chen et al, 2000). However, the structure and our expression experiments lead us to think of H9b as an integral component of the mNBD1 fold; at a minimum, the association is favorable.

\section{Preparation, crystallization, and structure determination of triphospho-mNBD1}

Phosphorylation of CFTR, particularly in the R domain, is thought to regulate channel opening and closing, and protein kinase A (PKA) plays an important role in this process (Ostedgaard et al, 2001). There are 21 PKA phosphorylation motifs (Kennelly and Krebs, 1991) in human CFTR. About 
one-quarter of these putative phosphorylation sites are located in mNBD1 (Ser422, Ser489, Ser519, Ser557, Ser660, and Ser670), and the murine sequence presents an additional PKA site at Ser659. To identify CFTR NBD1 residues available for PKA phosphorylation in vitro, we incubated mNBD1 with PKA and analyzed the results using phosphopeptide mapping by mass spectrometry. Residues Ser422, Ser659, Ser660, and Ser670 were seen to be phosphorylated with Ser659 to a lesser extent of only about one-quarter.

The phosphorylated form of the protein, mNBD1-P, crystallized isomorphously with the unmodified protein and we were able to determine its structure. Those portions of mNBD1 that are well-ordered in the unphosphorylated state are unchanged in the mNBD1-P structure, but the presence of phosphorserine 422 confers order on residues 420-428 (solid red ribbon segment in Figure 3D) in the inserted loop where a phosphate group is visible on the side chain of Ser422. In addition, phosphorylation is evident at residues Ser660 and Ser670, and partially at Ser659 (only seen in molecule A in the asymmetric unit). Phosphorylation at Ser422 (Chang et al, 1993), Ser660 (Cheng et al, 1991; Winter and Welsh, 1997), and Ser670 (Wilkinson et al, 1997) has been observed in human CFTR and each has been shown to have a specific effect on the activity of CFTR. Based on this evidence as well as discussions on the relevance of these regions to a putative NBD1-NBD2 heterodimer (see below), we will refer to the S1-S2 loop and H9c structures as the regulatory insertion and regulatory extension, respectively.

\section{Nucleotide coordination in mNBD1 crystal structures}

Nucleotides in the various crystal complexes with mNBD1 (Table I) are all bound in a very similar manner. This mode of binding has aspects in common with that in other $\mathrm{ABC}$ transporter NBDs, but the comparison is distinguished as much by differences as similarities. Since Mg-ATP has its $\gamma$-phosphate intact when cocrystallized with mNBD1, whether phosphorylated or not, mNBD1 is not an active ATPase as is typical for such domains. Moreover, since the protein conformation is the same when uncomplexed or complexed with ADP as when complexed with ATP (r.m.s.d. $=0.2-0.3 \AA$ ), the possibility is raised that nucleotide binding to the NBD1 site in CFTR is not involved directly in movements thought to drive $\mathrm{ABC}$ transporter action in other cases (Yuan et al, 2001).

Canonical features of nucleotide binding in mNBD1 all involve interactions with the phosphate groups (Figure 4A). Specifically, Lys464 and Thr465 (Walker A), Asp572 (Walker B), and Gln493 (Q-loop) hydrogen bond with the phosphates and/or coordinate the $\mathrm{Mg}^{2+}$ ion as in other $\mathrm{ABC}$ domain structures. On the other hand, consistent with a catalytically inactive site, there are also significant differences. Namely, the Walker-B carboxylate residue, typically glutamate, that serves as the catalytic base in active $\mathrm{ABC}$ transporters (Moody et al, 2002) is replaced by serine in NBD1 (Ser573), and the canonical H-loop histidine residue becomes Ser605. Both of these fail to hydrogen bond with the $\gamma$-phosphate as in related complexes (Hung et al, 1998; Smith et al, 2002).

The distinction from usual nucleotide binding by $A B C$ domains is even more pronounced for ribose and base interactions in mNBD1. The ATP ribose is $\mathrm{C}^{\prime}$-endo with an 'anti' $\chi$ torsion angle base conformation as in other ATPbound ABC structures (eg MJ0796 (Smith et al, 2002) and HisP (Hung et al, 1998)), but the ribose-base assembly is rotated away due to a backbone $\gamma$ torsion angle of gauche + as opposed to trans, which is seen in other $\mathrm{ABC}$ domain structures (Figure 4B). As a result, the adenine base of the nucleotide does not stack against an aromatic side chain. Instead, the position of the usual stacking aromatic residue (Tyr11 in MJ0796) is taken up by Trp401, which is in close contact with the ATP ribose ( $3.3 \AA$ ). Phe 430 , which is in the S1-S2 insertion, makes an 'edge-to-face' interaction with the adenine base. Leu409 makes contacts approaching from the opposite side of the ring. No hydrogen bonding contacts are observed with the adenine.

In the phosphorylated structure, mNBD1-P, residues 420 428 contribute additional contacts to the adenine base of the nucleotide (Figure 4C). Val428 is in van der Waals contact with the adenine as is Glu425, which stacks partially underneath the base. Together with Phe430, these residues serve to grip the nucleotide base. A hydrogen bond between the side chain of His421 and the phosphate of Ser660 contributes to the conformational stabilization of this region.

\section{ATP binding and hydrolysis in solution}

ATP binding to mNBD1 had an apparent $K_{\mathrm{D}}$ of $149 \pm 48 \mu \mathrm{M}$ (Figure 5A). Previously measured values for nucleotide affinity have ranged from low micromolar (Yike et al, 1996; Qu et al, 1997) to several millimolar (Ko et al, 1993), varying with both the nucleotide analog used as well as the protein construct. The values reported here are consistent with those of human NBD1 determined by several other groups (Yike et al, 1996; Qu et al, 1997; Neville et al, 1998). ATP binding was significantly reduced by the K464A mutation, presumably because electrostatic interactions with the $\beta$ - and $\gamma$-phosphates of ATP (Figure 4A) are lost.

ATP hydrolysis by mNBD1 in solution was measured in a standard coupled assay system (Rosing et al, 1975). An active ABC NBD ATPase, MJ0796, was included as a positive control. Consistent with previous experiments (Moody et al, 2002), the MJ0796 protein hydrolyzed ATP at a rate approaching $8 \mathrm{~min}^{-1}$, while the mNBD1 exhibited a hydrolytic rate less than $0.02 \mathrm{~min}^{-1}$ in the colorimetric assays in both the unphosphorylated and phosphorylated states (Figure 5B). The disparity with previously published results (Ko and Pedersen, 1995; Duffieux et al, 2000; Annereau et al, 2003) may reflect differences in ATP binding between mouse and human proteins or, more likely, are due to other factors associated with alternative constructs.

\section{Discussion}

\section{Intrinsic structural integrity}

The crystal structures of mNBD1 have features that distinguish them from other $\mathrm{ABC}$ cassette proteins. The structure is essentially the same independent of nucleotide state, nucleotides bind in an unusual conformation at the ribose-phosphate linkage, and regulatory segments are added to the core structure. Since mNBD1 molecules associate into head-to-tail tetramers in our crystals, we have considered whether these lattice contacts ( $\sim 900 \AA^{2}$ from each surface) might affect the intrinsic structure. The predominant interactions involve residues from the Q-loop and $\alpha$-subdomain at the 'head' of one molecule contacting H1c residues near the ATP site at the 'tail' of another molecule.

While lattice influences cannot be ruled out, several lines of evidence suggest that they do not overwhelm the intrinsic 
A

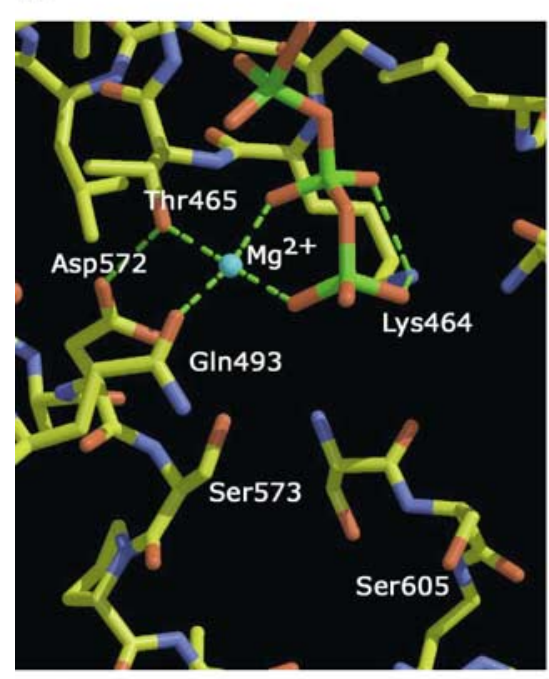

B

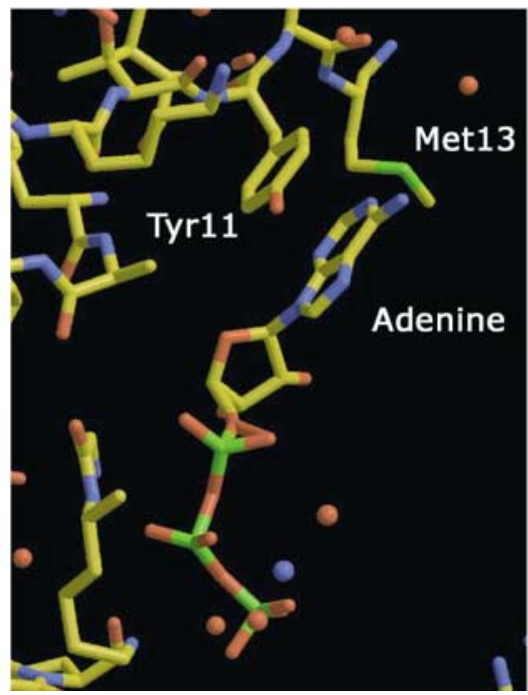

C
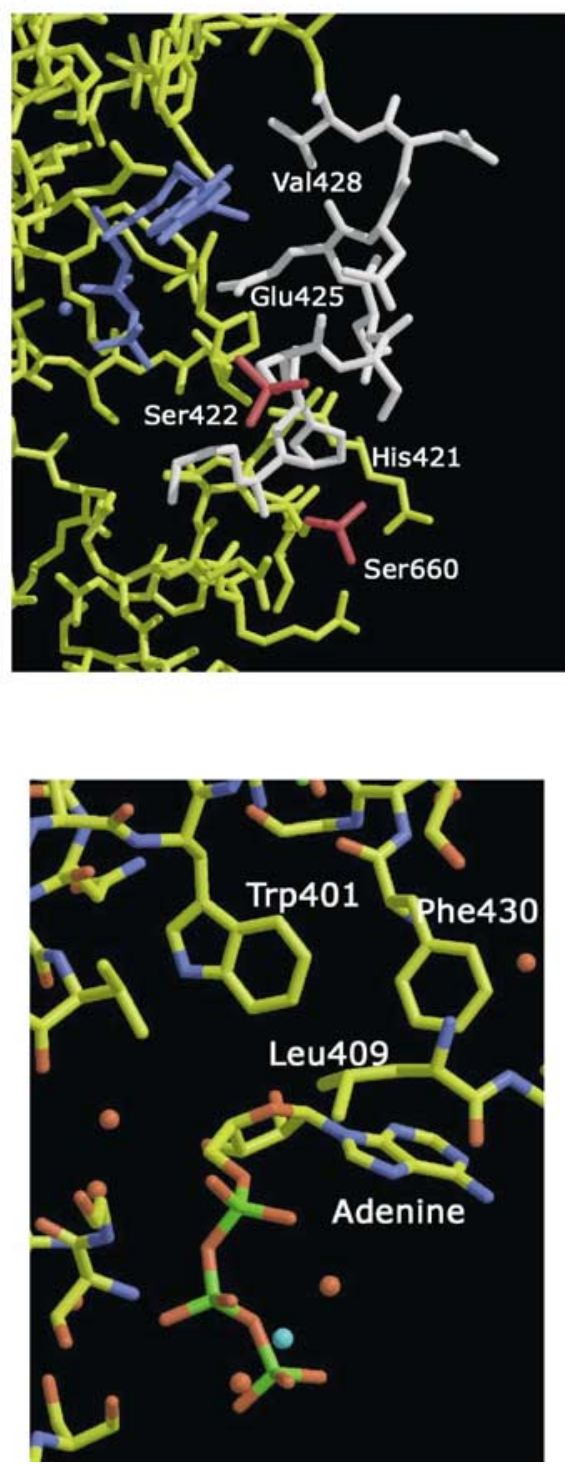

Figure 4 Close-up on ATP binding by mNBD1. (A) Canonical hydrogen bonding interactions in Mg-ATP mNBD1. Some relevant hydrogen bonds are indicated as green lines. Some residues in the foreground and background have been removed to clarify the interactions, here and in (B) and (C). (B) Differences in adenine base recognition from other ABC domains. Left: adenine stacks against Tyr11 of MJ0796 (PDB ID code 1L2T). Right: adenine of ATP makes edge-to-face interactions with Phe430 of mNBD1. (C) Added structure in phosphorylated mNBD1. The ATP molecule plus magnesium in blue, the additional mNBD1 residues observed in the phosphorylated state in white, the phosphate atoms of Ser422 and Ser660 in brown, and the remainder of the mNBD1 structure in yellow are shown.

mNBD1 structure. With respect to the relative disposition of $\mathrm{ABC} \alpha$ - and F1-ATPase subdomains, two facts are most compelling: (1) The same $\mathrm{I}_{1} 22$ crystals grow spontaneously and equally well from mNBD1 in Mg-ATP, Mg-ADP, and nucleotide-free states. (2) Intramolecular interactions between $\alpha$ - and F1-subdomains in mNBD1 (five hydrogen bonds and 34 residue pairs in van der Waals contact) are much more extensive than in the head-to-tail interface (one hydrogen bond and 16 van der Waals pairs). With respect to the unusual ribose-base conformation of nucleotides in mNBD1, this is largely determined by the placement of Trp401 in the S1-H1b segment, which is not involved in any contacts.

\section{Structural implications}

We expect NBD1 domains from all CFTRs to have essentially the same structure as that seen here for mNBD1. The known sequences of CFTR NBD1 from diverse vertebrate species are very similar (61-98\%, pairwise identity) and divergent segments are restricted to surface residues and loop regions. This suggests that all NBD1s share the deviations from canonical $\mathrm{ABC}$ domains that distinguish mNBD1. In particular, human NBD1 should be very close in structure to mNBD1 as these molecules are $78 \%$ identical in sequence over the span of mNBD1 and there are no amino-acid insertions or deletions (Figure 2A). It seems likely that its structure will also be similar in intact CFTR, but functional interactions with the NBD2, MSD1, and/or R domains of CFTR (Figure 1) might well lead to adaptations in NBD1.

Indications of potential plasticity in NBD1 come from the crystallographic measures of atomic mobility in mNBD1. Parts of the regulatory insertion between S1 and S2 are sufficiently flexible that they are not seen in the density 
A

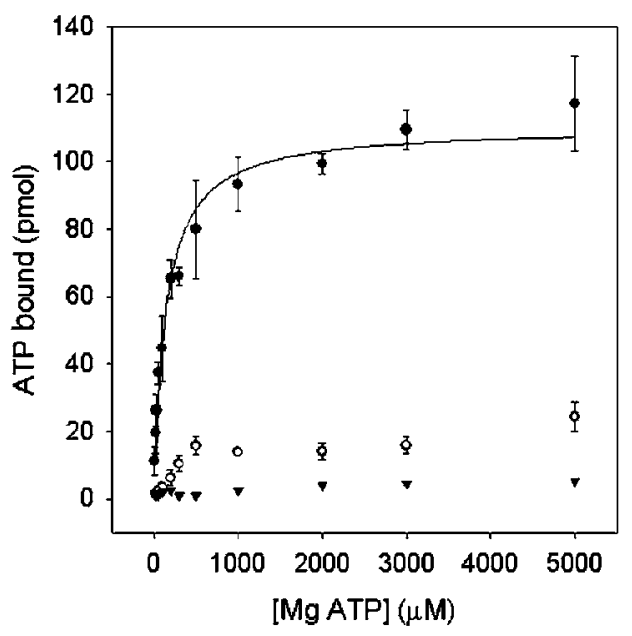

B

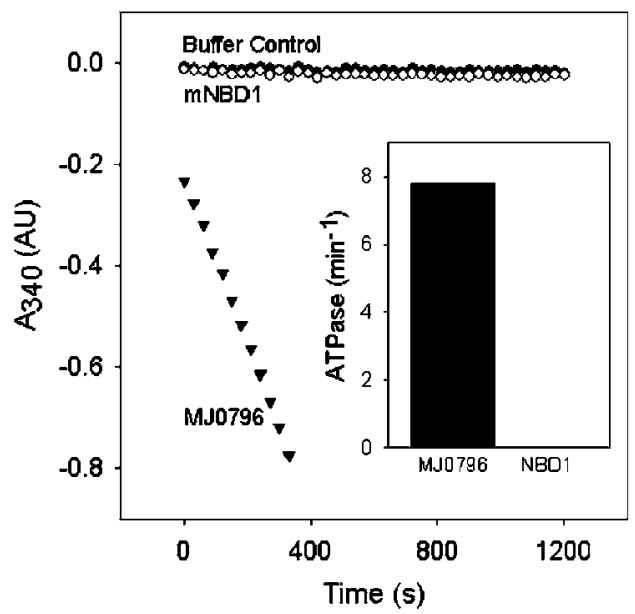

Figure 5 ATP binding and hydrolysis by mNBD1. (A) ATP binding to mNBD1 was analyzed using a filter-binding assay. Wild type $(\bullet)$, K464A mutant $(O)$, and ATP binding to the filters in the absence of protein $(\boldsymbol{\nabla})$ are shown. The wild-type $K_{\mathrm{D}}$ was calculated to be $149 \pm 48 \mu \mathrm{M}$. (B) ATPase activity was analyzed by a coupled colorimetric assay. ATPase activity for mNBD1, MJ0796 (LolD), and background are shown as a function of changes in NADH absorbance at $340 \mathrm{~nm}$. Inset: ATP hydrolysis rates were calculated to be less than $0.02 \mathrm{~min}^{-1}$ for the mNBD1, as compared to the positive control, MJ0796, which was $7.9 \mathrm{~min}^{-1}$.

maps and B-factors are elevated for the ordered parts, H1b and H1c, of the insertion (Figure 3B). Similarly, B-factors are also well above average for the helices H8-H9b. These elements are candidates for displacement by interactions with other CFTR domains or other regulatory events. Evidence that this may indeed happen comes in comparing our results with results from the coexpression of severed CFTR constructs (Chan et al, 2000). Wild-type conductance levels were observed from two-chain CFTR channels constructed such that either 415-432 (the flexible loop in our structures) or 634-667 (H8-H9b region) was omitted, suggesting that these features may not be vital for CFTR function. We were unable to generate stable NBD1 proteins from constructs any narrower than the ordered confines of
mNBD1 (approximately residues 390-670), but the NBD1 core may well be stabilized by other interactions in situ. NBD1 phosphorylation sites are located in these 'deletable' segments, and perhaps they can be displaced in intact CFTR when phosphorylated such that exposed surfaces can interact productively with other domains, notably NBD2.

\section{Enzymatic activity}

Our results demonstrate that mNBD1 is incapable, on its own, of catalyzing significant ATP hydrolysis: ATP remains intact in mNBD1 crystal structures, and no significant hydrolysis is observed in our solution assays. This lack of activity is not unexpected from the model espoused in Figure 1 whereby NBD1-NBD2 'heterodimers' are thought to mediate normal catalysis in CFTR, and likely only do so at the site where NBD2 provides the Walker A and B sequences (NBD2-based site). The catalytic base, usually glutamate, in the NBD1based site is replaced by serine at position 573 , and this putative site has other defects as well. We now know from the structures that there are no candidate replacements within NBD1 for the catalytic base. On the other hand, since the phosphate groups of ATP are otherwise coordinated in a conventional manner in mNBD1, the $\gamma$-phosphate is poised for attack and it is conceivable that some unanticipated part of CFTR might confer catalytic competence. However, NBD1 in intact CFTR does not appear to be enzymatically active (Basso et al, 2003; Vergani et al, 2003).

\section{Interactions between NBD1 and NBD2}

Our working hypothesis for the role of NBDs in CFTR function is that NBD1 interacts with NBD2 in the mode of a labile nucleotide sandwich, as depicted in Figure 1; ATP binding to the NBDs, and hydrolysis at NBD2, then couples through NBD-MSD associations to control the channel. This model is consistent with biochemical and structural information on homodimeric $\mathrm{ABC}$ transporter systems (Locher et al, 2002; Moody et al, 2002; Smith et al, 2002; Chen et al, 2003). It is also consistent with 'heterodimeric' crosslinking between NBD1 and NBD2 in human multidrug-resistant P glycoprotein (Loo et al, 2002) and with tryptophan-fluorescence assays of direct interactions for human CFTR between NBD1-R (373859), a construct that encompasses the entire span of our mNBD1, and NBD2 (1151-1476) (Wang et al, 2002). Finally, it is consistent with electrophysiological studies of CFTR function (Vergani et al, 2003), which indicate that normal channel opening requires Mg-ATP binding at both nucleotide sites and that normal channel closing follows ATP hydrolysis in the NBD2-based site. In order to pursue the hypothesis, we developed homology models for CFTR NBD2 and for the NBD1-NBD2 'heterodimer'.

We have docked our mNBD1 structure and our NBD2 homology model into a hypothetical NBD1-NBD2 'heterodimer' constructed with reference to the structure of the E171Q mutant MJ0796 homodimer (Smith et al, 2002). This model, in what is expected to be the universal dimerization mode of $\mathrm{ABC}$ transporters, exhibits severe main-chain steric clashes between NBD1 and NBD2 (Figure 6). Specifically, the inserted loop region (405-436) and C-terminal $\alpha$-helix (H9b) are thrust directly into the opposing NBD2 model. There are several ways in which this conflict might be resolved. One possibility is that there is a conformational change in NBD1 upon interdomain association, whereby the clashing regions 


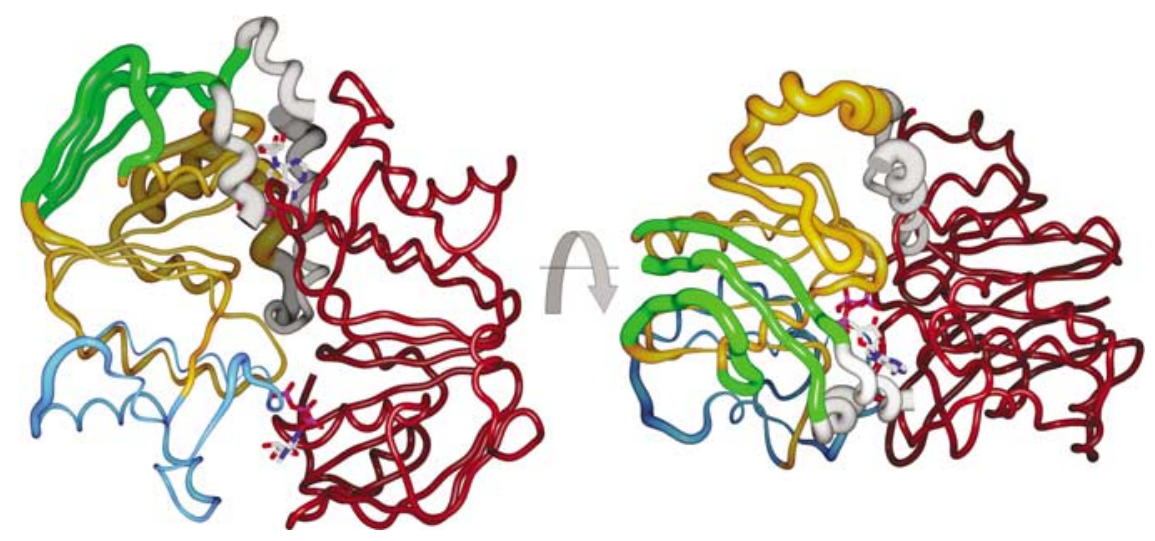

Figure 6 Worm figure of putative NBD1-NBD2 interaction. NBD1 is color coded as in Figure 2B. NBD2 is in red. The NBD1 worm thickness is proportional to the backbone B-factor. The figure at the right is rotated $90^{\circ}$ toward the viewer relative to the left figure.

are moved out of the way. The inserted loop and H9b extension have elevated B-factors (Figures $3 \mathrm{~B}$ and 6) and the correspondingly greater atomic mobilities may indicate hinged conformational flexibility. Moreover, sites of phosphorylation in mNBD1-P are all located on the S1-S2 insertion and on the H9b extension (Figure 3D), and these clashing segments might possibly be displaced when phosphorylated to take up favorable interactions with other parts of CFTR, thereby promoting and maintaining NBD association. This hypothesis is consistent with data from R-domain deletions, which release CFTR gating inhibitions upon phosphorylation (Rich et al, 1991) to give opening kinetics similar to phosphorylated wild-type CFTR but with less stable open channels (Csanady et al, 2000).

\section{Interactions between NBD1 and MSD1}

Associations between the NBD and MSD domains are expected to provide the conduit of communication from nucleotide-dependent conformational changes in NBD domains to the gating of transmembrane channels in $\mathrm{ABC}$ transporters. The structures of other $\mathrm{ABC}$ transporters (Chang and Roth, 2001; Locher et al, 2002; Chang, 2003) provide an insight into the nature of these associations. In each case, $\alpha$-domain and Q-loop portions of an NBD interact with cytoplasmic extensions from the transmembrane portion of one MSD-NBD exclusively. We constructed homology models of CFTR MSD1-NBD1 based on our structure of mNBD1 docked onto the corresponding region of the MsbA and BtuCD structures (not shown). The surface of NBD1 that would be at the interface with mMSD1 overlaps with a surface that is buried into the head-to-tail rings in mNBD1 crystal lattices. This surface includes the hydrophobic side chains from Phe494, Trp496, Met498, Val540, and Phe508, the most common CF mutation site.

\section{CF mutations in NBD1}

The majority of sites of CF-causing missense mutations occur in NBD1, primarily in its $\alpha$-subdomain, and the locations in the mNBD1 structure of the most common of these (A455E, G480C, I506T, $\Delta \mathrm{I} 507, \Delta \mathrm{F} 508, \mathrm{~S} 549 \mathrm{~N}, \mathrm{~S} 549 \mathrm{R}, \mathrm{G} 551 \mathrm{D}, \mathrm{A} 559 \mathrm{~T}$, R560T, Y569D, and D648V; Bobadilla et al, 2002) are shown in Figure 3D. The deletion of Phe508, $\Delta \mathrm{F} 508$, is by far the most prevalent. This mutation leads to reduced CFTR transport to, and retention in, the plasma membrane (Cheng et al,
1990), perhaps due to improper folding (Qu and Thomas, 1996) and instability of CFTR (Lukacs et al, 1993), which is detected by the protein degradation machinery in the endoplasmic reticulum (ER) and lysosomes (Gelman and Kopito, 2002). The limited number of $\Delta \mathrm{F} 508$ molecules that are retained form active chloride channels, but with altered gating properties (Dalemans et al, 1991).

Phe508 lies exposed on the surface of mNBD1, positioned at the start of a loop between helices H3 and H4. This site is distant ( $>20 \AA$ ) from either ATP in the 'heterodimer' model, suggesting that $\triangle \mathrm{F} 508$ is unlikely to affect ATP binding directly. The side chain of Phe508 is in the vicinity of Arg560, Trp496, and Met498, but it makes only a single van der Waals contact, with Met498. Thus, Phe508 does not appear to play a critical role in stabilizing mNBD1 structure. Moreover, its main-chain contacts are only local, so accommodation of the deletion through loop reorganization seems plausible. Disruptions are expected to be substantial nevertheless. One possible impact of $\Delta$ F508 might be on accessibility to the di-acidic code proposed to be involved in membrane protein transport out of the ER (Barlowe, 2003). Another impact may be on the NBD1-MSD1 interface into which Phe508 is expected to be buried (see above). CF mutations concentrate in the $\alpha$-subdomain or contacting sites, and disruptive consequences are evident from the structure for some.

\section{Materials and methods}

\section{Protein preparation and phosphorylation}

Mouse CFTR NBD1 (residues 389-673; human numbering) was expressed in $E$. coli as an $\mathrm{N}$-terminal $\mathrm{His}_{6}-\mathrm{Smt} 3$ fusion protein (Mossessova and Lima, 2000) using a pET26b-derived expression vector. Cells were harvested by centrifugation and lysed by sonication. Protein was initially purified by nickel ion affinity chromatography, followed by proteolytic removal of the tag using Ulp1 and GFC. A final nickel ion affinity step was employed to remove traces of the $\mathrm{His}_{6}-\mathrm{Smt} 3$ affinity tag. Final yields averaged $4 \mathrm{mg} / \mathrm{l}$ with greater than $98 \%$ purity. The purified protein was concentrated to $5-10 \mathrm{mg} / \mathrm{ml}$ in buffer containing $150 \mathrm{mM} \mathrm{NaCl}$, $12.5 \%$ glycerol, $2 \mathrm{mM}$ DTT, $2 \mathrm{mM}$ ATP, and $50 \mathrm{mM}$ Tris $\mathrm{pH}$ 7.5. SeMet containing protein was expressed and purified using similar procedures.

To generate phosphorylated mNBD1, $1000 \mathrm{U}$ of bovine PKA (Sigma P2645-1KU) was incubated with $5 \mathrm{mg}$ of mNBD1 at a concentration of $1 \mathrm{mg} / \mathrm{ml}$ at $4{ }^{\circ} \mathrm{C}$ for $4 \mathrm{~h}$, and then concentrated to $5-10 \mathrm{mg} / \mathrm{ml}$. Mass spectrometric characterization verified phos- 
phorylation of three or four sites (full modification: Ser422, Ser660, and Ser670; partial, approximately $25 \%$ modification: Ser659).

\section{Protein characterization}

Matrix-assisted laser desorption ionization and electrospray ionization mass spectrometric analyses confirmed the identity of the purified proteins and estimated purity at greater than $95 \%$. DLS measurements using a DynaPro-MS800 instrument made at protein concentrations up to $8 \mathrm{mg} / \mathrm{ml}$ showed a single monomeric species. Analytical gel filtration at a protein concentration of $4 \mathrm{mg} / \mathrm{ml}$ yielded similar results.

\section{Crystallization, data collection, structure determination, and refinement}

Purified $\mathrm{mNBD} 1$ at $5-10 \mathrm{mg} / \mathrm{ml}$ was subjected to initial hangingdrop vapor-diffusion screens with more than 500 different precipitant conditions at two temperatures $\left(4\right.$ and $\left.20^{\circ} \mathrm{C}\right)$. From this it was found that a simple precipitant consisting of $3.5-4.0 \mathrm{M}$ sodium acetate with no additional buffer or salt and $\mathrm{pH}$ adjusted to 7.5 yielded diffraction-quality crystals in two forms (I4,22: $a=140 \AA, \quad c=280 \AA$, two molecules/asymmetric unit; $\quad \mathrm{P}_{2} 22_{1} 2$ : $a=172 \AA, c=110 \AA$, four molecules/asymmetric unit). Nucleotide complexes were generated through cocrystallization, except in the case of ADP in which soaking of mNBD1 crystals with $1 \mathrm{mM}$ nucleotide was employed. Crystals were frozen by immersion in liquid nitrogen prior to data collection. Diffraction data were measured at the SGX-CAT beamline 31-ID at the Advanced Photon Source under standard cryogenic conditions, and processed with either the Mosflm (CCP4, 1994) or HKL (Otwinowski and Minor, 1997) software.

The structure was initially determined in space group $\mathrm{I}_{1} 22$ using the MIRAS method. Two $\mathrm{Ta}_{6} \mathrm{Br}_{12}$ derivative data sets, diffracting to $4.0 \AA$, were collected at the peak energy of tantalum and used with a native $3.1 \AA$ data set in phasing calculations. Cluster sites located with SNB (Weeks and Miller, 1999) gave phasing powers in MLPHARE (CCP4, 1994) of 2.12 and 1.98 for the two derivatives and produced experimental phases useful to $4.0 \AA$. Two copies of a homology model of NBD1 based on the TAP1 structure (PDB code $1 \mathrm{JJ} 7)$ were manually placed in the density-modified (DM; CCP4, 1994) electron density map. Further density modification with NCS averaging then produced better maps and allowed a more extensive NBD1 backbone construction.

The mNBD1 backbone from the $\mathrm{I}_{1} 22$ structure was positioned by molecular replacement into the $\mathrm{P} 42_{1} 2$ lattice using a $2.9 \AA$ data set acquired at the selenium peak energy of an SeMet crystal. A Bijvoet-difference Fourier based on resulting model phases allowed the identification of 21 Se sites, which were refined with MLPHARE using $2.5 \AA$ native $\mathrm{P} 42_{1} 2$ data. The resulting phases were NCS averaged to obtain a final map that was used for building the side chains and finalizing the model. The structure of NBD1 was built manually over several cycles of model building with XtalView (McRee, 1999) and refinement with CNX (Brunger et al, 1998) and/ or Refmac (CCP4, 1994). Subsequent structures were determined using the molecular replacement program EPMR (Kissinger et al, 1999), and refined to convergence. PROCHECK (Laskowski et al, 1993) revealed main-chain and side-chain structural parameters consistently better than average (overall $G$-values $>1.0$ ). See Table I for data collection and refinement statistics.

\section{ATP binding to NBD1}

ATP binding was measured using a standard filter-binding assay (Widlak et al, 2001). Endogenous ATP added during purification was first removed by exchanging (three times) the protein into

\section{References}

Annereau JP, Ko YH, Pedersen PL (2003) Cystic fibrosis transmembrane conductance regulator: the $\mathrm{NBF} 1+\mathrm{R}$ (nucleotide-binding fold 1 and regulatory domain) segment acting alone catalyses a $\mathrm{Co}^{2+} / \mathrm{Mn}^{2+} / \mathrm{Mg}^{2+}$-ATPase activity markedly inhibited by both $\mathrm{Cd}^{2+}$ and the transition-state analogue orthovanadate. Biochem $\mathrm{J}$ 371: 451-462

Barlowe C (2003) Signals for COPII-dependent export from the ER: what's the ticket out? Trends Cell Biol 13: 295-300

Basso C, Vergani P, Nairn AC, Gadsby DC (2003) Prolonged nonhydrolytic interaction of nucleotide with CFTR's $\mathrm{NH}_{2}$-terminal buffer lacking ATP (50 mM Tris- $\mathrm{HCl} \mathrm{pH} 7.6,150 \mathrm{mM} \mathrm{NaCl}, 2 \mathrm{mM}$ DTT, $12.5 \%$ glycerol) using Bio-Rad Micro Bio-Spin 6 chromatography columns following the manufacturer's protocols. mNBD1 protein $(375 \mathrm{pmol})$ was incubated with increasing concentrations of an $\mathrm{Mg}-\alpha^{32} \mathrm{P}$-ATP mixture for $45 \mathrm{~min}$ at room temperature in a final reaction volume of $20 \mu \mathrm{l}$. The protein was then applied to Millipore HA $0.45 \mu \mathrm{m}$ filters that were prewetted with buffer on a 12-position Millipore vacuum manifold. The samples were washed three times with $2 \mathrm{ml}$ of ice-cold buffer supplemented with $40 \mathrm{mM} \mathrm{MgCl}_{2}$ and then counted on a Beckman LS-6500 scintillation counter.

\section{ATP hydrolysis by NBD1}

ATP hydrolysis by mNBD1 was measured using a colorimetric assay described previously (Rosing et al, 1975). ATP hydrolysis was measured via a coupled reaction where NADH is converted to $\mathrm{NAD}^{+}$in a one-to-one ratio with ADP production. The reaction was monitored by changes in absorbance at $340 \mathrm{~nm}$. In total, $1 \mathrm{nmol}$ mNBD1 was incubated in reaction buffer $(100 \mathrm{mM}$ Tris $\mathrm{pH} 8.0$ $50 \mathrm{mM} \mathrm{KCl}, 2 \mathrm{mM} \mathrm{MgCl}$, $0.2 \mathrm{mM}$ EDTA, $4 \mathrm{mM}$ Mg-ATP, $1 \mathrm{mM}$ PEP, $200 \mu \mathrm{M} \mathrm{NADH}, 4 \mathrm{U} / \mathrm{ml} \mathrm{LDH}, 8 \mathrm{U} / \mathrm{ml} \mathrm{PK}$ ) in a final volume of $1.015 \mathrm{ml}$ at $30^{\circ} \mathrm{C}$ and monitored by changes in $\mathrm{A}_{340}$ on a Shimadzu UV2101 spectrophotometer. MJ0796 was used as a positive control for ATP hydrolysis. The rates of ATP hydrolysis were calculated as a function of the changes in $A_{340}$ using $\varepsilon^{\mathrm{NADH}}=6.22 \mathrm{mM} / \mathrm{cm}$.

\section{Homology modeling}

Homology models were built using SCWRL3 (Bower et al, 1997) and Modeller 6v2 (Fiser et al, 2000). Based on the assumption that CFTR NBD1 and NBD2 can interact, a heterodimer model was created using CFTR mNBD1 as a template for each domain and several NBD structures as templates for the dimer interaction, including BtuD (1L7V) and MJ0796 (1L2T). The Walker A, LSGGQ, and Walker B motifs of NBD1 were structurally aligned with each ABC monomer using Spock (Christopher, 1998), and then CFTR NBD1/NBD2 was modeled simultaneously. Multiple models were generated and the best model was chosen based on Modeller's energy term, ProsaII analysis (Sippl, 1993), and visual inspection.

\section{Figure preparation}

Structure figures were made with MOLSCRIPT (Kraulis, 1991) and Raster3D (Merritt and Bacon, 1997), except for Figures 3C, 4A, 4B and 4C which were made using Xtalview (McRee, 1999) and Figure $3 \mathrm{~B}$ and 6 which were made using Spock.

\section{Data deposition}

Coordinates have been deposited in the Protein Data Bank (http:// www.rcsb.org/pdb). Accession codes are listed in Table I.

\section{Acknowledgements}

We thank Drs E de la Fortelle and T Harris for many useful discussions; Dr A Verkman for supplying putative CFTR ligands used in some cocrystallization efforts; L Pelletier, F Lu, K Bain, and Drs S Antonysamy, IK Feil, J Hendle, B Noland, and F Park for their expert contributions towards the expression, characterization, and crystallization of mNBD1; and $\mathrm{K}$ Schwinn for his help in making some of the figures. Use of the Advanced Photon Source was supported by the US Department of Energy, Office of Science, and Office of Basic Energy Sciences, under Contract No. W-31-109-Eng38. This work was supported by a research contract from the Cystic Fibrosis Foundation. 
cidence data and application to screening. Hum Mutat 19: 575-606

Bower MJ, Cohen FE, Dunbrack Jr RL (1997) Prediction of protein side-chain rotamers from a backbone-dependent rotamer library: a new homology modeling tool. J Mol Biol 267: 1268-1282

Brunger AT, Adams PD, Clore GM, DeLano WL, Gros P, GrosseKunstleve RW, Jiang JS, Kuszewski J, Nilges M, Pannu NS et al. (1998) Crystallography \& NMR system: a new software suite for macromolecular structure determination. Acta Crystallogr D 54: 905-921

CCP4 (1994) The CCP4 suite: programs for protein crystallography. Acta Crystallogr D 50: 760-763

Chan KW, Csanady L, Seto-Young D, Nairn AC, Gadsby DC (2000) Severed molecules functionally define the boundaries of the cystic fibrosis transmembrane conductance regulator's $\mathrm{NH}(2)$ terminal nucleotide binding domain. J Gen Physiol 116: 163-180

Chang G (2003) Structure of MsbA from Vibrio cholera: a multidrug resistance $\mathrm{ABC}$ transporter homolog in a closed conformation. J Mol Biol 330: 419-430

Chang G, Roth CB (2001) Structure of MsbA from E. coli: a homolog of the multidrug resistance ATP binding cassette $(\mathrm{ABC})$ transporters. Science 293: 1793-1800

Chang XB, Tabcharani JA, Hou YX, Jensen TJ, Kartner N, Alon N, Hanrahan JW, Riordan JR (1993) Protein kinase A (PKA) still activates CFTR chloride channel after mutagenesis of all 10 PKA consensus phosphorylation sites. J Biol Chem 268: 11304-11311

Chen J, Lu G, Lin J, Davidson AL, Quiocho FA (2003) A tweezerslike motion of the ATP-binding cassette dimer in an ABC transport cycle. Mol Cell 12: 651-661

Chen JM, Scotet V, Ferec C (2000) Definition of a 'functional R domain' of the cystic fibrosis transmembrane conductance regulator. Mol Genet Metab 71: 245-249

Cheng SH, Gregory RJ, Marshall J, Paul S, Souza DW, White GA, O'Riordan CR, Smith AE (1990) Defective intracellular transport and processing of CFTR is the molecular basis of most cystic fibrosis. Cell 63: 827-834

Cheng SH, Rich DP, Marshall J, Gregory RJ, Welsh MJ, Smith AE (1991) Phosphorylation of the $\mathrm{R}$ domain by cAMP-dependent protein kinase regulates the CFTR chloride channel. Cell 66: 1027-1036

Christopher JA (1998) SPOCK: The Structural Properties Observation and Calculation Kit. Program Manual College Station, TX: A\&M University

Csanady L, Chan KW, Seto-Young D, Kopsco DC, Nairn AC, Gadsby DC (2000) Severed channels probe regulation of gating of cystic fibrosis transmembrane conductance regulator by its cytoplasmic domains. J Gen Physiol 116: 477-500

Dalemans W, Barbry P, Champigny G, Jallat S, Dott K, Dreyer D, Crystal RG, Pavirani A, Lecocq JP, Lazdunski M (1991) Altered chloride ion channel kinetics associated with the delta F508 cystic fibrosis mutation. Nature 354: $526-528$

Duffieux F, Annereau JP, Boucher J, Miclet E, Pamlard O, Schneider M, Stoven V, Lallemand JY (2000) Nucleotide-binding domain 1 of cystic fibrosis transmembrane conductance regulator production of a suitable protein for structural studies. Eur J Biochem 267: 5306-5312

Fiser A, Do RK, Sali A (2000) Modeling of loops in protein structures. Protein Sci 9: 1753-1773

Gelman MS, Kopito RR (2002) Rescuing protein conformation: prospects for pharmacological therapy in cystic fibrosis. $J$ Clin Invest 110: 1591-1597

Hopfner KP, Karcher A, Shin DS, Craig L, Arthur LM, Carney JP, Tainer JA (2000) Structural biology of Rad50 ATPase: ATP-driven conformational control in DNA double-strand break repair and the ABC-ATPase superfamily. Cell 101: 789-800

Hung LW, Wang IX, Nikaido K, Liu PQ, Ames GF, Kim SH (1998) Crystal structure of the ATP-binding subunit of an ABC transporter. Nature 396: 703-707

Karpowich N, Martsinkevich O, Millen L, Yuan YR, Dai PL, MacVey K, Thomas PJ, Hunt JF (2001) Crystal structures of the MJ1267 ATP binding cassette reveal an induced-fit effect at the ATPase active site of an ABC transporter. Structure (Camb) 9: 571-586

Kennelly PJ, Krebs EG (1991) Consensus sequences as substrate specificity determinants for protein kinases and protein phosphatases. J Biol Chem 266: 15555-15558
Kissinger CR, Gehlhaar DK, Fogel DB (1999) Rapid automated molecular replacement by evolutionary search. Acta Crystallogr D 55: 484-491

Ko YH, Pedersen PL (1995) The first nucleotide binding fold of the cystic fibrosis transmembrane conductance regulator can function as an active ATPase. J Biol Chem 270: 22093-22096

Ko YH, Thomas PJ, Delannoy MR, Pedersen PL (1993) The cystic fibrosis transmembrane conductance regulator. Overexpression, purification, and characterization of wild type and delta F508 mutant forms of the first nucleotide binding fold in fusion with the maltose-binding protein. J Biol Chem 268: 24330-24338

Kraulis PJ (1991) MOLSCRIPT: a program to produce both detailed and schematic plots of protein structures. J Appl Crystallogr 24: 946-950

Laskowski RJ, MacArthus MW, Moss DS, Thornton JM (1993) PROCHECK: aprogram to check stereochemical quality of protein structures. J Appl Crystallogr 26: 283-291

Locher KP, Lee AT, Rees DC (2002) The E. coli BtuCD structure: a framework for $\mathrm{ABC}$ transporter architecture and mechanism. Science 296: 1091-1098

Loo TW, Bartlett MC, Clarke DM (2002) The 'LSGGQ' motif in each nucleotide-binding domain of human P-glycoprotein is adjacent to the opposing walker A sequence. J Biol Chem 277: 4130341306

Lukacs GL, Chang XB, Bear C, Kartner N, Mohamed A, Riordan JR, Grinstein $S$ (1993) The $\Delta$ F508 mutation decreases the stability of cystic fibrosis transmembrane conductance regulator in the plasma membrane. Determination of functional half-lives on transfected cells. J Biol Chem 268: 21592-21598

McRee DE (1999) XtalView/Xfit-a versatile program for manipulating atomic coordinates and electron density. J Struct Biol 125: 156-165

Merritt EA, Bacon DJ (1997) Raster3D photorealistic molecular graphics. Methods Enzymol 277: 505-524

Moody JE, Millen L, Binns D, Hunt JF, Thomas PJ (2002) Cooperative, ATP-dependent association of the nucleotide binding cassettes during the catalytic cycle of ATP-binding cassette transporters. J Biol Chem 277: 21111-21114

Mossessova E, Lima CD (2000) Ulp1-SUMO crystal structure and genetic analysis reveal conserved interactions and a regulatory element essential for cell growth in yeast. Mol Cell 5: 865-876

Neville DC, Rozanas CR, Tulk BM, Townsend RR, Verkman AS (1998) Expression and characterization of the NBD1-R domain region of CFTR: evidence for subunit-subunit interactions. Biochemistry 37: 2401-2409

Ostedgaard LS, Baldursson O, Welsh MJ (2001) Regulation of the cystic fibrosis transmembrane conductance regulator $\mathrm{Cl}$-channel by its R domain. J Biol Chem 276: 7689-7692

Ostedgaard LS, Rich DP, DeBerg LG, Welsh MJ (1997) Association of domains within the cystic fibrosis transmembrane conductance regulator. Biochemistry 36: 1287-1294

Otwinowski Z, Minor W (1997) Processing of X-ray diffraction data collected in oscillation mode. Methods Enzymol 276: 307-326

Qu BH, Strickland EH, Thomas PJ (1997) Localization and suppression of a kinetic defect in cystic fibrosis transmembrane conductance regulator folding. $J$ Biol Chem 272: 15739-15744

Qu BH, Thomas PJ (1996) Alteration of the cystic fibrosis transmembrane conductance regulator folding pathway. J Biol Chem 271: 7261-7264

Riordan JR, Rommens JM, Kerem B, Alon N, Rozmahel R, Grzelczak Z, Zielenski J, Lok S, Plavsic N, Chou JL et al (1989) Identification of the cystic fibrosis gene: cloning and characterization of complementary DNA. Science 245: 1066-1073

Rosing J, Harris DA, Kemp Jr A, Slater EC (1975) Nucleotide-binding properties of native and cold-treated mitochondrial ATPase. Biochim Biophys Acta 376: 13-26

Schmitt L, Benabdelhak H, Blight MA, Holland IB, Stubbs MT (2003) Crystal structure of the nucleotide-binding domain of the ABC-transporter Haemolysin B: identification of a variable region within ABC helical domains. J Mol Biol 330: 333-342

Schmitt L, Tampe R (2002) Structure and mechanism of ABC transporters. Curr Opin Struct Biol 12: 754-760

Sippl MJ (1993) Recognition of errors in three-dimensional structures of proteins. Proteins 17: 355-362

Smith PC, Karpowich N, Millen L, Moody JE, Rosen J, Thomas PJ, Hunt JF (2002) ATP binding to the motor domain from an ABC 
transporter drives formation of a nucleotide sandwich dimer. Mol Cell 10: 139-149

Verdon G, Albers SV, Dijkstra BW, Driessen AJM, Thunnissen AWH (2003) Crystal structures of the ATPase subunit of the blucose ABC transporter from Sulfolobus solfataricus: nucleotide-free and nucleotide-bound conformations. J Mol Biol 330: 343-358

Vergani P, Nairn AC, Gadsby DC (2003) On the mechanism of MgATP-dependent gating of CFTR $\mathrm{Cl}(-)$ channels. J Gen Physiol 121: $17-36$

Wang W, He Z, O’Shaughnessy TJ, Rux J, Reenstra WW (2002) Domain-domain associations in cystic fibrosis transmembrane conductance regulator. Am J Physiol Cell Physiol 282: C1170-C1180

Weeks CM, Miller R (1999) Optimizing shake-and-bake for proteins. Acta Crystallogr D 55: 492-500

Widlak P, Li LY, Wang X, Garrard WT (2001) Action of recombinant human apoptotic endonuclease $\mathrm{G}$ on naked DNA and chromatin substrates: cooperation with exonuclease and DNase I. J Biol Chem 276: 48404-48409

Wilkinson DJ, Strong TV, Mansoura MK, Wood DL, Smith SS, Collins FS, Dawson DC (1997) CFTR activation: additive effects of stimulatory and inhibitory phosphorylation sites in the $\mathrm{R}$ domain. Am J Physiol 273: L127-L133

Winter MC, Welsh MJ (1997) Stimulation of CFTR activity by its phosphorylated R domain. Nature 389: 294-296

Yike I, Ye J, Zhang Y, Manavalan P, Gerken TA, Dearborn DG (1996) A recombinant peptide model of the first nucleotide-binding fold of the cystic fibrosis transmembrane conductance regulator: comparison of wild-type and delta F508 mutant forms. Protein Sci 5: 89-97

Yuan YR, Blecker S, Martsinkevich O, Millen L, Thomas PJ, Hunt JF (2001) The crystal structure of the MJ0796 ATP-binding cassette. Implications for the structural consequences of ATP hydrolysis in the active site of an $\mathrm{ABC}$ transporter. J Biol Chem 276: $32313-32321$ 